- Case Report

\title{
Exertional Rhabdomyolysis after Spinning
}

\author{
Youjin Jeong, Hyuk-Jung Kweon*, Eun-Jung Oh, Ah-Leum Ahn, Jae-Kyung Choi, Dong-Yung Cho \\ Department of Family Medicine, Konkuk University School of Medicine, Seoul, Korea
}

\begin{abstract}
Any strenuous muscular exercise may trigger rhabdomyolysis. We report an episode of clinically manifested exertional rhabdomyolysis due to stationary cycling, commonly known as spinning. Reports of spinning-related rhabdomyolysis are rare in the English literature, and the current case appears to be the first such case reported in South Korea. A previously healthy 21-year-old Asian woman presented with severe thigh pain and reddish-brown urinary discoloration 24-48 hours after attending a spinning class at a local gymnasium. Paired with key laboratory findings, her symptoms were suggestive of rhabdomyolysis. She required hospital admission to sustain renal function through fluid resuscitation therapy and fluid balance monitoring. Because exertional rhabdomyolysis may occur in any unfit but otherwise healthy individual who indulges in stationary cycling, the potential health risks of this activity must be considered.
\end{abstract}

Keywords: Rhabdomyolysis; Stationary Cycling; Acute Kidney Injury 


\section{INTRODUCTION}

Rhabdomyolysis may occur when muscles are overworked. ${ }^{1)}$ Although several cases of exercise-related rhabdomyolysis have been documented, exertional rhabdomyolysis due to stationary cycling is seldom reported. Indoor cycling fitness, commonly known as spinning, is a high-intensity exercise involving a stationary bicycle. Originating in California around $1989,{ }^{2)}$ this trend has gained international exposure. Thus, a wave of rhabdomyolysis due to spinning is not unexpected along with its recent increase in popularity. There are few reported instances of rhabdomyolysis in this setting, with only ten similar episodes published in the English scientific literature. ${ }^{1-5)}$ To our knowledge, the case presented here is the first reported Korean case of spinning-induced rhabdomyolysis.

\section{CASE REPORT}

In June 2014, a 21-year old female Asian college student who was previously healthy but unfit presented with bilateral thigh pain and dark red-colored urine. Two days earlier, she had participated in a supervised spinning class, completing the 40-minute session without any noticeable discomfort. Despite mild post-workout limb soreness, she attempted a second session on the following day. Twenty minutes after the class started, her condition deteriorated. Worsening pain and weakness in both anterior thighs forced her to withdraw from the session. Later that evening, she noted variable brown urinary discoloration. She claimed to have been well-hydrated during the workout and indicated that she did not wear a rubber suit.

Her medical history was noncontributory. She denied intake of dietary supplements or drugs, specifically oral contraceptives and use of cigarettes or alcohol. No family history of neuromuscular or renal disease was elicited. A review of her systems revealed no abnormal findings (i.e., no fever, malaise, or muscular weakness). Her vital signs were age-appropriate, and her body mass index was $24.5 \mathrm{~kg} / \mathrm{m}^{2}$. On physical examination, her thighs were tender and swollen, with restricted active and passive ranges of motion in hip and knee joints due to severe muscle pain. She was unable to fully extend her knees due to the pain.

Her laboratory values (with reference ranges) on the day of admission were: creatine phosphokinase (CPK), 16,370 IU/L (range, 20-200 IU/L); lactate dehydrogenase (LDH), 2,310 IU/L (range, 263-450 IU/L); aspartate aminotransferase (AST), 537 IU/L (range, 15-41 IU/L); and alanine transaminase (ALT), $160 \mathrm{U} / \mathrm{L}$ (range, 4-43 IU/L). Her renal function remained within normal limits (blood urea nitrogen [BUN], 8 $\mathrm{mg} / \mathrm{dL}$ [range, 8-26 mg/dL] and creatine [Cr], $0.5 \mathrm{mg} / \mathrm{dL}$ [range, 0.4$1.0 \mathrm{mg} / \mathrm{dL}])$. Urinalysis was negative for both myoglobin and hemoglobin. Her presenting history and laboratory findings confirmed a diagnosis of rhabdomyolysis. The patient was admitted for intravenous fluid resuscitation therapy and monitoring of fluid balance. Her CPK and liver enzyme levels declined thereafter, her pain diminished, and her urinary color returned to normal. At discharge on day 10, her laboratory values were: $\mathrm{CPK}, 460 \mathrm{IU} / \mathrm{L} ; \mathrm{LDH}, 459 \mathrm{IU} / \mathrm{L} ; \mathrm{BUN}, 10 \mathrm{mg} / \mathrm{dL}$; and $\mathrm{Cr}, 0.5 \mathrm{mg} / \mathrm{dL}$. At the one-week follow-up visit, her CPK (214 IU/L) and LDH (380 IU/L) levels were near normal (Table 1).

\section{DISCUSSION}

There are few published reports on rhabdomyolysis after spinning workouts. ${ }^{1-5)}$ However, primary care physicians should know the clinical features and potential complications in these circumstances. Delay in diagnosis or management may lead to life-threatening consequences. The clinical course of rhabdomyolysis may vary widely, ranging from asymptomatic illness to severe and debilitating sequelae. ${ }^{3,46)} \mathrm{Ex}$ ertional rhabdomyolysis should be suspected whenever a patient presents with vague symptoms of muscular pain and weakness after recent strenuous activity. ${ }^{5)}$

The major clinical symptoms of rhabdomyolysis, regardless of cause, include myalgia, muscular weakness, and dark-colored urine. ${ }^{7)}$ However, this classic triad develops in $<10 \%$ of patients. ${ }^{6,7)}$ Associated myoglobinuria, which results in urinary discoloration from a pinkish tinge to cola-colored or dark black urine, may lead to acute kidney injury in severe cases. ${ }^{7)}$ In addition, patients may present with constitutional symptoms such as fever, nausea, emesis, confusion, agitation, delirium, and anuria. ${ }^{7)}$

If historical and physical findings are consistent with rhabdomyolysis, the diagnosis may be confirmed by laboratory studies. Increased serum CPK levels are the most sensitive laboratory index of muscle injury. ${ }^{6)} \mathrm{CPK}$ levels at least 10 times the upper limit of the reference range (45-260 IU) are sufficient for diagnosis. ${ }^{6,7)}$ Typically, serum CPK levels rises within 12 hours of muscular injury, peaking in 1-3 days, and then declining over 3-5 days. There is no consensus on threshold CPK val-

Table 1. Laboratory results

\begin{tabular}{|c|c|c|c|c|c|c|}
\hline Day & $\begin{array}{c}\text { Creatine } \\
\text { phosphokinase (IU/L) }\end{array}$ & $\begin{array}{c}\text { Lactate } \\
\text { dehydrogenase (IU/L) }\end{array}$ & $\begin{array}{c}\text { Aspartate } \\
\text { aminotransferase (IU/L) }\end{array}$ & $\begin{array}{c}\text { Alanine transaminase } \\
(\mathrm{IU} / \mathrm{L})\end{array}$ & $\begin{array}{l}\text { Blood urea nitrogen } \\
\qquad(\mathrm{mg} / \mathrm{dL})\end{array}$ & $\begin{array}{c}\text { Creatinine } \\
\text { (mg/dL) }\end{array}$ \\
\hline Reference range & $5-200$ & $263-450$ & $15-41$ & $4-43$ & $8-26$ & $0.4-1.0$ \\
\hline 1st day (admission) & 16,370 & 2,310 & 537 & 160 & 8 & 0.5 \\
\hline 3rd day & 6,660 & & 289 & 184 & 8 & 0.5 \\
\hline 4th day & 3,319 & & 156 & 153 & & \\
\hline 6th day & 1,078 & & 65 & 103 & 8 & 0.6 \\
\hline 10th day (discharge) & 460 & 459 & 38 & 73 & 10 & 0.5 \\
\hline 1-Week follow-up & 214 & 380 & 33 & 41 & 7.4 & 0.6 \\
\hline
\end{tabular}


ues that signify a significant risk for renal failure. ${ }^{8)}$ Patients with peak CPK levels $<15,000-20,000$ IU on admission are generally at low risk of acute kidney injury, although CPK levels as low as 5,000 IU have been associated with renal failure ${ }^{6,8,9)}$ Myoglobin is often detected by urinalysis, with visible urinary discoloration at myoglobin concentrations $>100 \mathrm{mg} / \mathrm{dL}^{6,7)}$

The complications of rhabdomyolysis can be classified as early or late. Early complications include electrolyte disturbances (e.g., hyperkalemia and hypocalcemia) and elevated liver enzymes, whereas late complications include acute renal failure and disseminated intravascular coagulation, which develop 12-72 hours after injury. ${ }^{6)}$

Management of patients with rhabdomyolysis calls for early, aggressive replacement of fluids to support renal function. ${ }^{6,9)}$ Delay in rehydration may increase the risk of renal failure. Adequate early volume replacement within 6 hours of admission appears to minimize the risk of acute renal failure. ${ }^{6,7)}$ The prognosis of rhabdomyolysis depends on the nature of related complications and existing comorbid conditions. Early and aggressive treatment typically has an excellent prognosis. ${ }^{7,10)}$ The associated all-cause mortality rate is about $8 \%$ to $10 \%$.,10)

In addition to prompt diagnosis and treatment, prevention is also important. Individuals who lack prior fitness training, are dehydrated, or suffer heat stress are more prone to rhabdomyolysis. ${ }^{3)}$ In our patient, poor physical condition and abrupt, intensive exercise may have precipitated her episode. Hence, adequate hydration and proper fitness training to avoid muscular injury are recommended if strenuous physical activity, such as a spinning workout, is contemplated.

As reported in this care, exertional rhabdomyolysis may occur in any unfit but otherwise healthy individual who engages in stationary cycling, underscoring the potential health risk of this activity. Rhabdomyolysis was strongly suggested by her symptoms at presentation and the results of the physical examination. A careful, detailed history is necessary for diagnosis and timely treatment. Participants performing any rigorous physical training like spinning should be advised to maintain hydration and avoid undue strenuous exercise.

\section{CONFLICT OF INTEREST}

No potential conflict of interest relevant to this article was reported.

\section{REFERENCES}

1. Dawson J, Carl I. 'Too much, too soon,' or 'a step too far?' exercise induced Rhabdomyolysis. Ulster Med J 2013;82:39.

2. Parmar S, Chauhan B, DuBose J, Blake L. Rhabdomyolysis after spin class? J Fam Pract 2012;61:584-6.

3. Harper J, Wong E. Muscle soreness in a fit young woman: the patient exercised regularly, so what caused swelling and soreness after a single exercise class? [Internet]. Clin Advis 2009 May 4 [cited 2016 Mar 10]. Available from: http://www.clinicaladvisor.com/muscle-soreness-inafit-young-woman/article/136126/.

4. Inklebarger J, Galanis N, Kirkos J, Kapetanos G. Exercise-induced rhabdomyolysis from stationary biking: a case report. Hippokratia 2010;14:279-80.

5. Thoenes M. Rhabdomyolysis: when exercising becomes a risk. J Pediatr Health Care 2010;24:189-93.

6. Huerta-Alardin AL, Varon J, Marik PE. Bench-to-bedside review: rhabdomyolysis: an overview for clinicians. Crit Care 2005;9:158-69.

7. Zutt R, van der Kooi AJ, Linthorst GE, Wanders RJ, de Visser M. Rhabdomyolysis: review of the literature. Neuromuscul Disord 2014;24:651-9.

8. Brown CV, Rhee P, Chan L, Evans K, Demetriades D, Velmahos GC. Preventing renal failure in patients with rhabdomyolysis: do bicarbonate and mannitol make a difference? J Trauma 2004;56:1191-6.

9. Bosch X, Poch E, Grau JM. Rhabdomyolysis and acute kidney injury. N Engl J Med 2009;361:62-72.

10. Bagley WH, Yang H, Shah KH. Rhabdomyolysis. Intern Emerg Med 2007;2:210-8. 